\title{
TECNOLOGIA MINERAL PARA CINZAS DA COMBUSTÃO DE CARVÃO MINERAL DA REGIÃO CARBONÍFERA DO BAIXO JACUÍ-RS
}

\author{
Sydney Sabedot ${ }^{\prime}$ \\ Marcelo Garcia Sundstron ${ }^{2}$ \\ Gerson Luis Miltzarek ${ }^{3}$ \\ Carlos Hoffmann Sampaio ${ }^{4}$
}

\section{Resumo}

Este artigo reúne informações e dados sobre os aspectos físicos que marcam as cinzas de carvão mineral geradas na queima da matéria-prima em usinas térmicas. A abordagem se dá sob o foco da tecnologia mineral, com o objetivo de aproveitamento econômico dos resíduos e gestão ambiental sustentável. Os métodos utilizados relacionam-se com a distribuição granulométrica, colorimetria, densidade e moabilidade. Os principais resultados são: as cinzas pesadas, em geral, têm $D_{50}<0,6 \mathrm{~mm}$ e as cinzas leves $<20 \mu \mathrm{m}$; as alvuras em ambas as cinzas variam muito, indicando variações na presença de carvão incombusto nas amostras das três usinas; as densidades, em ambas as cinzas, variam entre 2,16 e $2,33 \mathrm{~g} / \mathrm{cm}^{3}$, indicando homogeneidade entre elas; o Índice de Trabalho de Bond em amostra de cinza pesada tem valor de II,9 kWh/t, indicando ser o material de fácil moagem. Com estes resultados de características físicas, conclui-se que, além das cinzas leves, as cinzas pesadas também podem ser aplicadas como aditivo à indústria do cimento, com o uso de processos de moagem de baixo custo operacional.

Palavras-chave: Tecnologia mineral; Cinza de carvão; Cinza pesada; Cinza leve.

\section{MINERAL TECHNOLOGY FOR COAL COMBUSTION ASHES FROM BAIXO JACUÍ-RS COAL AREA}

\begin{abstract}
This paper shows information and data about the physical characteristics of coal ash samples generated in power plants that burn coal. The study focuses on mineral technology and the objectives are both the economic uses and sustainable environmental management for the waste. The methods applied are related to particle size distribution, colorimetry, density and grindability. The main results are: bottom ash and fly ash generally have $D_{50}$, respectively, $<0.6 \mathrm{~mm}$ and $<20 \mu \mathrm{m}$; the brightness values in both ashes vary widely, indicating variations in the presence of unburned coal in the samples of three power plants; the densities in both ashes ranging from 2.16 to $2.33 \mathrm{~g} / \mathrm{cm}^{3}$, indicating homogeneity among them; the Bond Work Index value is $11.9 \mathrm{kWh} / \mathrm{t}$ in a sample of bottom ash, indicating that the material is easy to grinding. These results suggest that physical characteristics of the bottom ash, as the fly ash, also can be applied as an additive to the cement industry, using grinding processes with low operating cost.
\end{abstract}

Keywords: Mineral technology; Coal ash; Bottom ash; Fly ash.

\section{INTRODUÇÃO}

Este artigo aborda o tema cinzas pesadas e leves geradas em três usinas térmicas que queimam carvão mineral extraído na região do Baixo Jacuí, Estado do Rio Grande do Sul. Seu contexto está focado na tecnologia mineral, sob a qual os materiais estão sendo estudados e avaliados com o propósito de aproveitamento dos resíduos que retornam ao produtor da matéria-prima e acumulam nos seus pátios, caracterizando passivos ambientais.

Os estudos vêm se desenvolvendo há mais de três anos e constituem etapas sucessivas de pesquisas demandadas por

'Centro Universitário La Salle - UNILASALLE, Canoas, RS, Brasil. E-mail: sabedot@unilasalle.edu.br.

${ }^{2}$ Copelmi Mineração Ltda., Butiá, RS, Brasil.

${ }^{3}$ Universidade Federal do Rio Grande do Sul - UFRGS, Laboratório de Processamento Mineral, Porto Alegre, RS, Brasil.

${ }^{4}$ Universidade Federal do Rio Grande do Sul - UFRGS, Laboratório de Processamento Mineral, Porto Alegre, RS, Brasil. 
profissionais e entidades científicas e industriais interessados em definir métodos e rotas de tecnologia mineral para o aproveitamento econômico, especialmente das cinzas pesadas, visando definir processos operacionais suportados no conceito de desenvolvimento sustentável da mineração. Os estudos estão tendo suporte técnico, operacional e financeiro das entidades envolvidas e deverão continuar por cerca de mais dois anos com suporte financeiro do CNPq, através de projeto de pesquisa em desenvolvimento pelos autores do artigo.

O escopo completo da pesquisa foi definido em quatro etapas sequenciais: caracterização física das cinzas pesadas e leves; caracterização química e mineralógica das mesmas cinzas; definição de rota de beneficiamento mineral para as cinzas pesadas; e estudo de aproveitamento econômico do processo. O objetivo deste artigo é mostrar os estudos e resultados das amostragens 3 e 4 da primeira etapa da pesquisa, com o indicativo sobre o potencial para o aproveitamento dos resíduos quanto às suas características físicas. Os estudos e resultados das amostragens I e 2 encontram-se em Sabedot [I].

Segundo o DNPM [2], em 2012 o Brasil produziu $6,18 \times 10^{6}$ t de carvão mineral, cabendo ao Estado do Rio Grande do Sul cerca de 64\% da produção total. Na atualidade, a Copelmi Mineração Ltda., empresa participante neste estudo, é responsável por mais de $80 \%$ do carvão de uso industrial no País. A empresa extrai e beneficia carvão ROM na região do Baixo Jacuí, RS, gerando produtos diversificados que atendem especificações de clientes que geram energia pela queima destes produtos [3].

A queima de carvão mineral em usinas térmicas gera três resíduos sólidos: escória, cinza pesada (ou de fundo) e cinza leve (ou volante). Escória e cinza de fundo são partículas de dimensões variadas, geralmente grossas, que se acumulam em depósitos temporários nas empresas que queimam o carvão. Posteriormente, retornam à empresa geradora da matéria-prima, sendo depositadas em pilhas nas proximidades da mina, constituindo rejeito mineral. A cinza leve, constituída por partículas de dimensões inferiores a $0,15 \mathrm{~mm}$, é arrastada pelos gases da combustão e acumulada para posterior comercialização [4].

As cinzas leves são resíduos aproveitados há muito tempo como aditivo ao Cimento Portland Pozolânico pelas indústrias de cimento e concreto e, por isso, no RS, há muito pouco passivo ambiental decorrente deste resíduo. No entanto, as cinzas pesadas se acumulam nos pátios das mineradoras de carvão, potencializando impactos ambientais que preocupam os setores públicos e privados, no que tange à gestão ambiental em suas áreas.

Rohde [5] indica que as cinzas de carvão, longe de constituírem apenas resíduos da conversão termelétrica, correspondem a uma categoria de minério não-metálico que constituem o terceiro recurso mineral não-metálico do Estado do Rio Grande do Sul e o quarto em Santa Catarina, e que, como subprodutos da combustão de carvão, podem resultar em numerosos benefícios. Diversos são os usos para as cinzas do carvão: aterro e base para pavimentação em rodovias e em construções, obtenção de zeólitas, extração de metais, produtos cerâmicos especiais e produção de vidro, entre outros [6-10]. Muitos estudos, em diversos países, indicam que as cinzas pesadas com adequados parâmetros físicos, químicos e mineralógicos também podem ser incorporadas como aditivo às misturas da indústria cimenteira [1 I-16]. O presente artigo representa uma parte dos resultados da pesquisa, a qual está focada no aproveitamento das cinzas pesadas pela indústria cimenteira.

\section{MATERIAIS E MÉTODOS}

O carvão que originou as cinzas leves e pesadas do estudo pertencia aos depósitos carboníferos da região do Baixo Jacuí, RS, tendo sido extraído e beneficiado pela empresa Copelmi Mineração Ltda. Esta empresa fornece a matéria-prima para diversos clientes, dos quais foram escolhidas, aleatoriamente, para a amostragem, as empresas Tractebel, CMPC e Braskem, cuja coleta das cinzas ocorreu imediatamente após a queima do carvão em suas usinas. Neste estudo, as amostras destas empresas estão identificadas, respectivamente, como TRAC, CMPC e BRAS.

As amostras de ambas as cinzas, nas três usinas térmicas, foram coletadas em quatro etapas que representaram períodos de tempo distanciados entre si em torno de quatro meses, representando um ano de amostragem. No total, foram geradas 24 amostras, sendo 12 de cinzas pesadas e 12 de cinzas leves. Cada amostra pesou cerca de $20 \mathrm{~kg}$. Individualmente, foram homogeneizadas e quarteadas gerando as alíquotas utilizadas nos diversos estudos. Neste estudo, as cinzas leves e pesadas estão identificadas, respectivamente, como LEV e PES. E as quatro etapas de amostragem como AMI, AM2, AM3 e AM4.

As 24 amostras foram fisicamente caracterizadas quanto à distribuição granulométrica, colorimetria e densidade real. Uma amostra de cinza pesada foi submetida a um processo de moabilidade, que determina o Índice de Trabalho de Bond.

As análises para a distribuição granulométrica das cinzas pesadas foram realizadas no laboratório da Copelmi Mineração Ltda., conforme a norma ABNT NBR 7181:1984 [17]. Para as cinzas leves, as análises foram realizadas no Laboratório de Processamento Mineral (Laprom), da Universidade Federal do Rio Grande do Sul (UFRGS), em um granulômetro à difração laser Cilas, que determina a distribuição granulométrica de partículas de dimensões inferiores a 500 micra, em 50 frações.

Os ensaios de colorimetria foram realizados no Laprom, com o uso de um espectrocolorímetro Minolta. As amostras foram pulverizadas e constituíram pastilhas conforme a norma TAPPI T 534 pm-92 [18], e os procedimentos para a determinação do fator de reflectância ocorreram segundo a norma ABNT NBR NM-ISO 2470:200 I [19]. 
As determinações de densidade real ocorreram no Laboratório de Geotecnia da Associação Beneficente da Indústria Carbonífera de Santa Catarina (SATC), seguindo norma DNER-ME 093/94 [20].

O teste de moabilidade foi realizado no Laboratório de Tecnologia Mineral da COPPE/UFRJ em apenas uma amostra de cinza pesada da amostragem 2. Este teste é utilizado para o cálculo do Índice de Trabalho de Bond e foi realizado conforme a norma ABNT NBR I I376: 1990 [2 I].

\section{RESULTADOS}

\section{I Distribuição Granulométrica}

As Figuras I e 2 mostram as curvas de distribuição granulométrica das cinzas pesadas, respectivamente, das amostragens 3 e 4 , nas três usinas, e as Figuras 3 e 4 mostram as curvas das cinzas leves, nas mesmas amostragens.

\subsection{Colorimetria}

AMOSTRAS 3 - CINZA PESADA

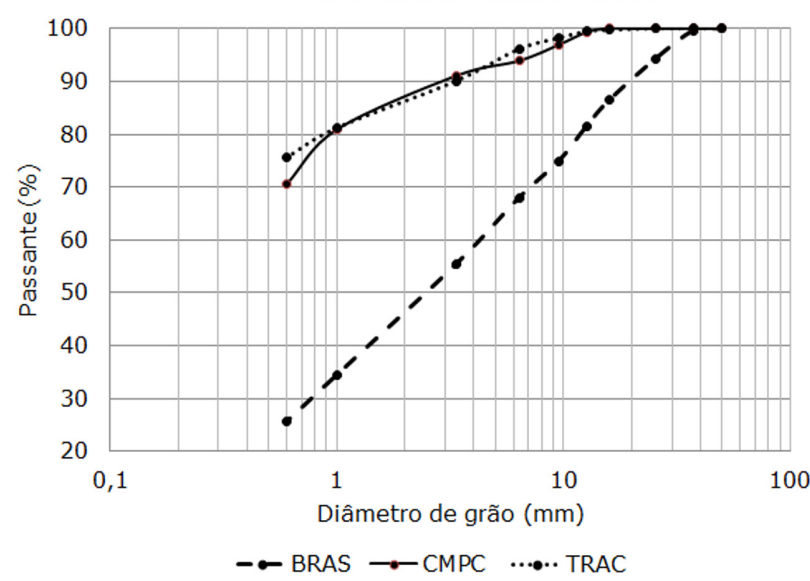

Figura I. Curvas granulométricas das cinzas pesadas da AM3.

AMOSTRAS 4 - CINZA PESADA

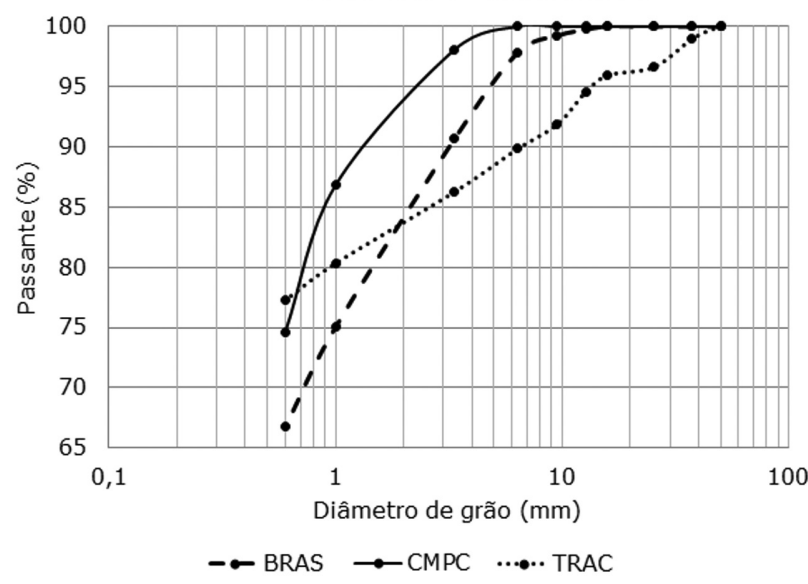

Figura 2. Curvas granulométricas das cinzas pesadas da AM4.
As Figuras 5 e 6 mostram as curvas de reflectância das cinzas pesadas, respectivamente, das amostragens 3 e 4 , e as Figuras 7 e 8 mostram as curvas das cinzas leves, nas mesmas amostragens.

A Tabela I mostra os valores dos parâmetros colorimétricos relacionados aos ensaios de colorimetria em ambas as cinzas, nas amostragens 3 e 4 , e os valores para uma amostra de carvão beneficiado que alimentou as fornalhas das três usinas.

\subsection{Densidade Real}

A Tabela 2 mostra valores para as densidades reais das cinzas coletadas nas amostragens 3 e 4 .

\section{4 Índice de Trabalho de Bond}

A Figura 9 mostra as curvas de distribuição granulométrica da amostra TRAC-PES-AM2 submetida ao teste de moabilidade e do produto da moagem após o teste.

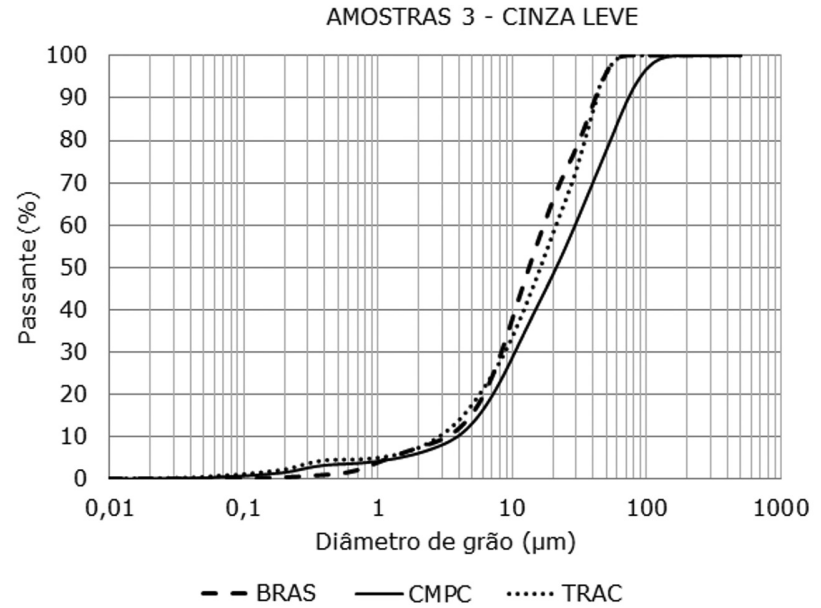

Figura 3. Curvas granulométricas das cinzas leves da AM3.

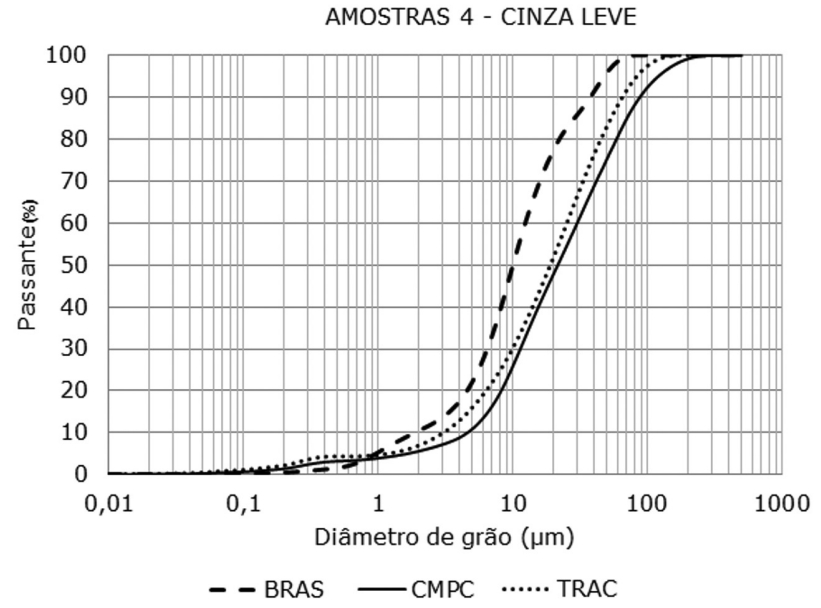

Figura 4. Curvas granulométricas das cinzas leves da AM4. 
AMOSTRAS 3 - CINZA PESADA

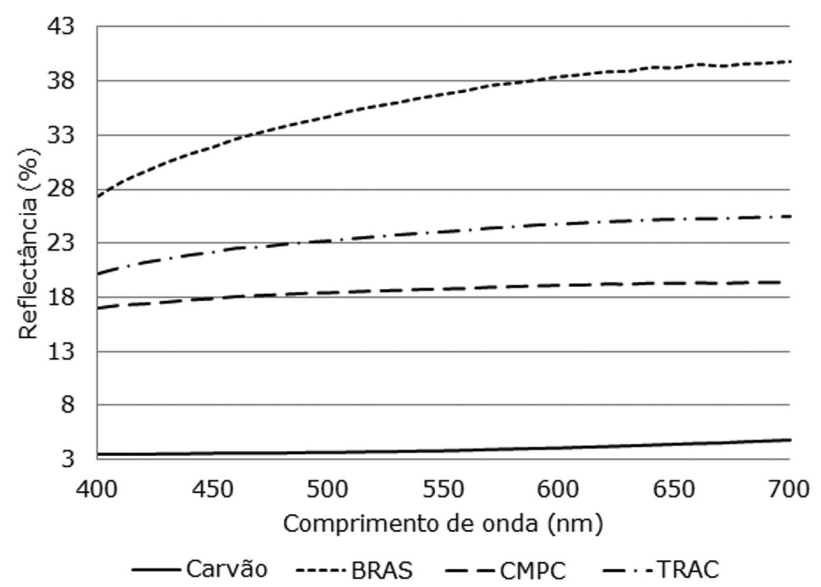

Figura 5. Curvas de reflectância das cinzas pesadas da AM3.

AMOSTRAS 4 - CINZA PESADA

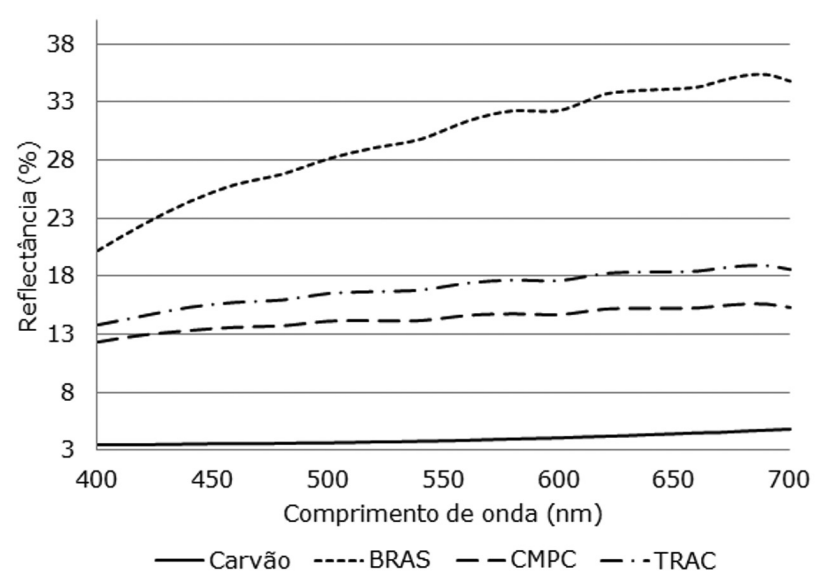

Figura 6. Curvas de reflectância das cinzas pesadas da AM4.

\section{DISCUSSÃO}

\section{I Distribuição Granulométrica}

As cinzas pesadas, observadas em estereomicroscópio trinocular, correspondem a um material agregado com minerais, cenosferas e carvão incombusto. Sua consistência é baixa, friável e se desfaz facilmente com a sua manipulação. Considerando a aplicação que se quer dar ao material, este fato é muito positivo, pois pode ser cominuído a um baixo custo operacional. $O$ teste de moabilidade, cuja discussão encontra-se adiante, mostrou esta viabilidade. Deste modo, os valores da distribuição granulométrica das cinzas pesadas podem variar dependendo de sua manipulação e da intensidade do peneiramento, parâmetros que foram foco de cuidados neste estudo.

Quanto aos dados das Figuras I e 2, observa-se que o material BRAS tende a concentrar na fração $>0,6 \mathrm{~mm}$ e apresentar distribuição mais homogênea nesta fração, enquanto que os materiais CMPC e TRAC tendem a concentrar na fração $<0,6 \mathrm{~mm}$ e distribuição heterogênea

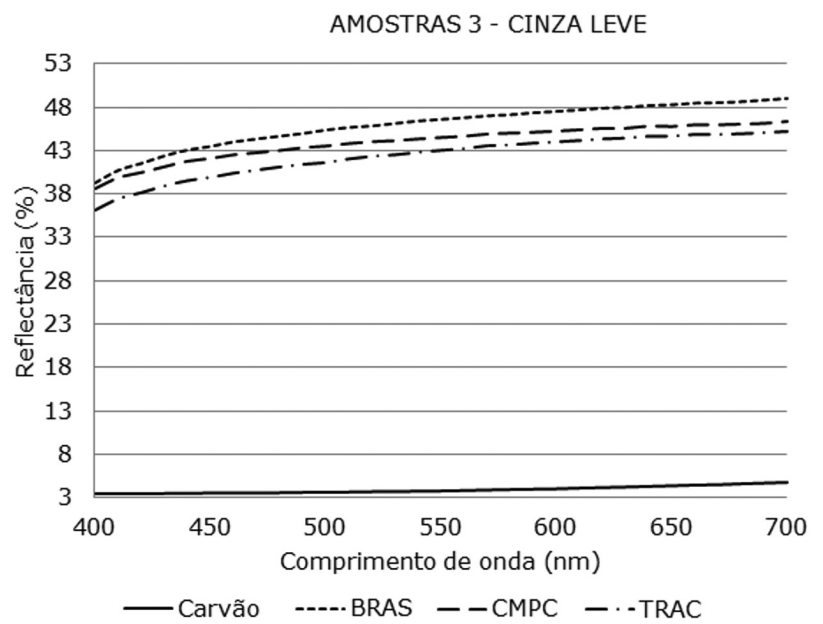

Figura 7. Curvas de reflectância das cinzas leves da AM3.

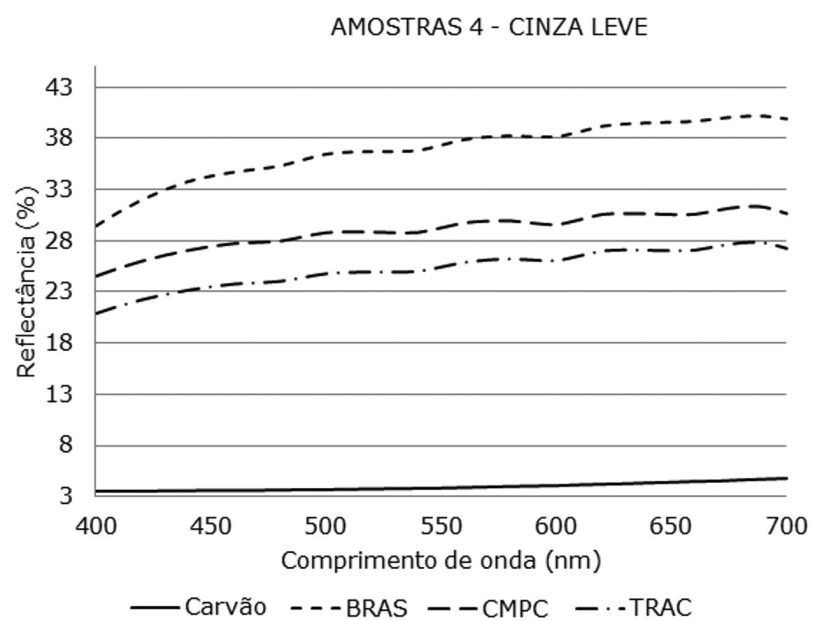

Figura 8. Curvas de reflectância das cinzas leves da AM4.

na fração $>0,6 \mathrm{~mm}$. Estes dados estão similares com os resultados das amostragens I e 2 definidos por Sabedot [I]. O conjunto de valores de todas as amostras de cinzas pesadas, associados à baixa consistência dos materiais avaliados, conferem características físicas favoráveis à moabilidade dos materiais gerados nas três empresas para eventual uso como matéria-prima na indústria cimenteira.

Quanto às cinzas leves, Figuras 3 e 4, os dados mostram similaridades quanto a valores granulométricos $e$ formatos de curvas, nas três empresas e nas duas amostragens. Observam-se valores para $D_{50}$ levemente mais baixos para as amostras BRAS e levemente mais altos para as amostras CMPC, o que confere, igualmente, para as cinzas leves, similaridade entre estas características e as mostradas por Sabedot [I] nas amostragens I e 2 .

\subsection{Colorimetria}

Estudos com colorimetria são comuns em materiais cuja cor desempenha fator decisivo na sua utilização como matéria-prima em misturas diversas. É o caso do caulim, 
Tabela I. Parâmetros colorimétricos das AM3 e AM4

\begin{tabular}{|c|c|c|c|c|c|c|c|}
\hline \multirow{2}{*}{ AM } & \multirow{2}{*}{ Parâmetro } & \multicolumn{2}{|c|}{ BRAS } & \multicolumn{2}{|c|}{ CMPC } & \multicolumn{2}{|c|}{ TRAC } \\
\hline & & PES & LEV & PES & LEV & PES & LEV \\
\hline \multirow[t]{4}{*}{3} & $\mathrm{~L}^{*}$ & 66,967 & 73,884 & 50,407 & 72,556 & 56,103 & 71,493 \\
\hline & $\mathrm{a}^{*}$ & 0,487 & 0,18 & 0,164 & 0,132 & 0,391 & 0,265 \\
\hline & $\mathrm{b}^{*}$ & 6,34 & 3,451 & I,777 & 2,837 & 3,205 & 3,56 \\
\hline & Alvura ISO & $32,25 \%$ & $43,76 \%$ & $17,99 \%$ & $42,31 \%$ & $22,37 \%$ & $40,19 \%$ \\
\hline \multirow[t]{4}{*}{4} & $\mathrm{~L}^{*}$ & 62,041 & 67,562 & 44,873 & 60,98 & 48,513 & 57,563 \\
\hline & $a^{*}$ & I,348 & 0,363 & 0,516 & 0,306 & 0,732 & 0,678 \\
\hline & $\mathrm{b}^{*}$ & 8,301 & 4,287 & 2,489 & 3,034 & 3,867 & 3,515 \\
\hline & Alvura ISO & $25,72 \%$ & $34,59 \%$ & $13,56 \%$ & $27,59 \%$ & $15,68 \%$ & $23,71 \%$ \\
\hline Carvão & L* & \multicolumn{6}{|c|}{23,167} \\
\hline \multirow[t]{3}{*}{ Beneficiado } & $a^{*}$ & \multicolumn{6}{|c|}{$\mathrm{I}, 258$} \\
\hline & $\mathrm{b}^{*}$ & \multicolumn{6}{|c|}{ I,79| } \\
\hline & Alvura ISO & \multicolumn{6}{|c|}{$3,56 \%$} \\
\hline
\end{tabular}

Tabela 2. Densidade real $\left(\mathrm{g} / \mathrm{cm}^{3}\right)$ das cinzas pesadas e leves das AM3 e AM4

\begin{tabular}{cccccccc}
\hline \multirow{2}{*}{ AM } & \multicolumn{2}{c}{ BRAS } & \multicolumn{3}{c}{ CMPC } & \multicolumn{2}{c}{ TRAC } \\
\cline { 2 - 8 } & PES & LEV & PES & LEV & PES & LEV \\
\hline 3 & 2,33 & 2,18 & 2,26 & 2,17 & 2,27 & 2,19 \\
4 & 2,32 & 2,16 & 2,25 & 2,16 & 2,18 & 22 \\
\hline
\end{tabular}

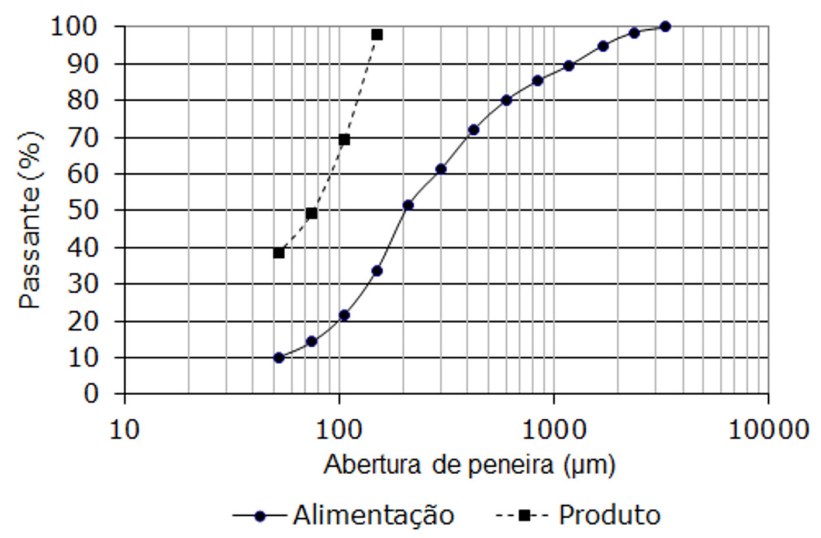

Figura 9. Curvas granulométricas de cinza pesada em teste de moabilidade.

cujos parâmetros colorimétricos definem se a substância pode ou não ter aplicação em misturas específicas. Não se encontrou publicação científica referenciando estudos de colorimetria com cinzas de carvão. Neste estudo, tenta-se verificar uma eventual correlação entre a quantidade de carvão incombusto nas cinzas e sua cor, através dos indicadores colorimétricos alvura ISO (brightness) e L*, a* e b*. Além disso, estes indicadores podem ser utilizados para definir o potencial das cinzas como matéria-prima para misturas com outras substâncias onde a cor seja importante. As cores dos materiais são definidas através dos padrões CIELAB. Neste processo, mede-se a intensidade de absorção da luz incidente, na região que compreende os comprimentos de onda do visível, e se obtêm os parâmetros L* (luminosidade, que varia do preto $(0)$ ao branco $(100)$ ), a* (intensidade da cor vermelho $(+)$ ou verde $(-))$ e b* (intensidade da cor amarelo $(+)$ ou azul (-)).

$\mathrm{Na}$ Tabela I, observa-se que o valor da alvura do carvão beneficiado é baixo (3,56\%), considerado normal para um material de cor quase preta. Para as cinzas pesadas, das três empresas, observa-se que a alvura é significativamente mais elevada, devido a eficácia do processo de queima, que gerou cinzas pesadas com baixo teor de carvão incombusto nas amostragens 3 e 4. Nas amostragens I e 2, no entanto, os valores de alvura das cinzas pesadas das empresas Braskem e CMPC variaram entre $5,82 \%$ e $8,79 \%$, o que indicava alto teor de carvão incombusto, consequência de problemas no processo de queima do carvão beneficiado [I]. Estes dados, mostrados aos técnicos das empresas, serviram como indicativos da necessidade de melhorias na performance da queima, realizadas e constatadas nos valores das amostragens 3 e 4. Quanto às cinzas leves, os valores das alvuras nas amostragens I, 2 e 3 são similares entre as três empresas, tendo sido constatado valores menores na amostragem 4 , nas três empresas, sem que se pudesse associar algum fato específico a respeito.

Nas Figuras 5 e 6, as curvas (ou os espectros) de reflectância das cinzas pesadas indicam que a Braskem teve a melhor performance de queima, devido a curva encontrar-se mais afastada da curva do carvão beneficiado, colocada no gráfico como referência. Comparativamente, a CMPC foi a empresa que apresentou performance de queima inferior no período. As Figuras 7 e 8 , correspondentes às cinzas leves, mostram curvas que também indicam que a Braskem teve melhor operação de queima de carvão no período, 
considerando-se que refletâncias mais altas são indicativas de menor quantidade de carvão incombusto.

Os dados colorimétricos não possibilitaram observar alguma relação entre os seus valores e o teor de material incombusto nas cinzas leves e pesadas. Pretende-se um estudo compartilhado com os resultados de análises químicas, para se verificar a possibilidade de alguma tendência.

\subsection{Densidade Real}

Os valores mostrados na Tabela 2 são muito semelhantes para ambas as cinzas das três empresas, sendo observado um pequeno aumento de valor nas cinzas pesadas. Os dados são igualmente semelhantes aos obtidos nas amostragens I e 2, por Sabedot [I]. Rohde [4] realizaram medições em amostras de duas usinas citadas neste estudo e os resultados foram similares. Segundo os autores, os valores de densidade real para as cinzas podem ser considerados como muito baixos se comparados aos valores dos solos em geral, devido a ocorrência de agregados e partículas nas cinzas que mantêm gases inclusos no seu interior.

\section{4 Índice de Trabalho de Bond}

Também conhecido como work index (WI), este índice é utilizado para materiais submetidos a processos de moagem em moinhos de bolas, segundo a terceira lei da cominuição, formulada por Bond [22]. Seus valores variam entre 5 e $25 \mathrm{kWh} / \mathrm{t}$. Numericamente, corresponde à energia total, expressa em $\mathrm{kWh}$ por tonelada curta $(907 \mathrm{~kg})$, necessária para reduzir o material, desde um tamanho teoricamente infinito até $80 \%$ passante em $106 \mu \mathrm{m}$ [23]. Segundo a lei de Bond, a energia para fragmentar a massa de um material homogêneo é inversamente proporcional à raiz quadrada do diâmetro das partículas [24].

A equação I é a expressão matemática da lei de Bond, onde: W é a energia em $\mathrm{kWh}$ para moer uma tonelada curta do material; WI é o índice de trabalho (work index); $\mathrm{P}$ é a abertura da peneira, em $\mu \mathrm{m}$, através da qual passam $80 \%$ da massa do produto; $\mathrm{F}$ é a abertura da peneira, em $\mu \mathrm{m}$, através da qual passam $80 \%$ da massa da alimentação [22].

$$
W=10 W I\left(\frac{1}{\sqrt{\mathrm{P}}}-\frac{1}{\sqrt{\mathrm{F}}}\right)
$$

A amostra de cinza pesada submetida ao teste de moabilidade indicou um valor de $1 \mathrm{I}, 9 \mathrm{kWh} / \mathrm{t}$ para $\mathrm{WI}$, o qual pode ser considerado como médio. $O$ consumo energético da moagem deste material é, por exemplo, inferior ao necessário à moagem de clínquer de cimento Portland, e superior à matéria-prima para cimento $[1,23]$.

\section{CONCLUSÕES}

As amostras foram coletadas em periodicidades regulares durante um ano, tempo considerado suficiente para denotar representatividade. A comparação de resultados nos quatro procedimentos de coleta mostra que os valores pouco diferem entre si nas amostras de cada empresa, permitindo concluir que, mantidas as operações, novas amostragens e análises tenderão a indicar resultados semelhantes aos mostrados neste estudo.

Considerando o objetivo para a aplicação das cinzas pesadas na indústria do cimento, as características físicas, químicas e mineralógicas devem ser avaliadas. Os indicadores físicos do presente estudo mostraram resultados que indicam viabilidade técnica para o objetivo proposto, sendo animadores para a sequência da pesquisa. A distribuição granulométrica das cinzas pesadas pode ficar adequada por moagem de baixo custo, considerando-se a condição friável do material à fricção ou impacto, bem como o resultado do teste de moabilidade, que indicou um valor de $1 \mathrm{I}, 9 \mathrm{kWh} / \mathrm{t}$ para $\mathrm{WI}$, o qual indica baixo consumo energético na moagem do material, para deixá-lo com a granulometria adequada àquela indústria.

\section{Agradecimentos}

Os autores agradecem às empresas citadas no presente estudo, por disponibilizarem acesso às instalações e funcionários na coleta e tratamento de amostras; ao CNPq, que concedeu e concede auxílio financeiro ao primeiro autor, através dos processos 475682/2009-2 e 471590/2013-4.

\section{REFERÊNCIAS}

I Sabedot S, Sundstron MG, Böer SC, Sampaio CH, Dias RGO. Caracterização e aproveitamento de cinzas da combustão de carvão mineral geradas em usinas termelétricas. In: Anais do III Congresso Brasileiro de Carvão Mineral; 20II ago 21-24; Gramado, Brasil.

2 Brasil. Ministério de Minas e Energia. Departamento Nacional de Produção Mineral - DNPM. Sumário Mineral 20 I3. Brasília; 2013. v. 33.

3 Copelmi Mineração Ltda. Produzindo desenvolvimento com responsabilidade socioambiental. [acesso em 28 out. 2014]. Disponível em: http://www.copelmi.com.br. 
Sabedot et al.

4 Rohde GM, Zwonok O, Chies O, Silva NLW. Cinzas de carvão fóssil no Brasil: aspectos técnicos e ambientais. Porto Alegre: CIENTEC; 2006.

5 Rohde GM. Cinzas, a outra metade do carvão fóssil-nova estratégia para a termeletricidade. In: Anais do IV Congresso Brasileiro de Carvão Mineral; 2013 ago 22-24; Gramado, Brasil. p. 40-54.

6 Santos W. Avaliação da cinza pesada gerada no Complexo Termoelétrico Jorge Lacerda para uso em aterros de trecho da duplicação da BR IOI [monografia]. Tubarão: Universidade do Sul de Santa Catarina; 2008.

7 Ryu TG, Ryu JC, Choi CH, Kim CG, Yoo SJ, Yang HS, et al. Preparation of Na-PI zeolite with high cation exchange capacity from coal fly ash. Journal of Industrial and Engineering Chemistry. 2006; I 2:40 I-407.

8 Suárez-Ruiz I, Hower JC, Thomas GA. Hg and Se capture and fly ash carbons from combustion of complex pulverized feed blends mainly of anthracitic coal rank in Spanish power plants. Energy \& Fuels. 2007;2I(I):59-70. http://dx.doi.org/l0.102I/ef060348I.

9 Ozdemir O, Ersoy B, Celik MS. Separation of pozzolonic material from lignitic fly ash of Tuncbilek Power Station. In: Proceedings of the 200I International Ash Utilization Symposium; 200I October 22-24. Lexington: University of Kentucky. p. 216-234.

10 Erol M, Küçükbayrak S, Ersoy-Meriçboyu A. Characterization of coal fly ash for possible utilization in glass production. Fuel. 2007;86(5-6):706-7I4. http://dx.doi.org/I0.1016/j.fuel.2006.09.009.

I I American Coal Ash Association - ACAA. Coal combustion products: beneficial use: simply recycling by another name. Aurora: American Coal Ash Association Educational Foundation; 2009.

12 Andrade LB, Rocha JC, Cheriaf M. Influence of coal bottom ash as fine aggregate on fresh properties of concrete. Construction \& Building Materials. 2009;23(2):609-6/4. http://dx.doi.org/10.1016/j.conbuildmat.2008.05.003.

13 Kochert S, Ricci D, Sorrenti R, Bertolino M. Transforming bottom ash into fly ash in coal fired power stations. World of Coal Ash Conference; 2009; Lexington, USA.

14 Kurama H, Kaya M. Usage of coal combustion bottom ash in concrete mixture. Construction \& Building Materials. 2008;22(9):1922-1928. http://dx.doi.org/10.1016/j.conbuildmat.2007.07.008.

15 Andrade LB. Metodologia de avaliação para uso de cinzas pesadas de termelétrica para agregado de concreto [dissertação]. Florianópolis: UFSC; 2004.

16 Kreuz AL. Utilização de cinzas pesadas de termelétricas na substituição de cimento e areia na confecção de concretos [dissertação]. Florianópolis: UFSC; 2002.

17 Associação Brasileira de Normas Técnicas - ABNT. NBR 7I8I: solo: análise granulométrica: método de ensaio. Rio de Janeiro; 1984.

18 Technical Association of the Pulp and Paper Industry - TAPPI. T 534 pm-92: brightness of clay and other mineral. Pigments (d/0 diffuse). Atlanta; 1994.

19 Associação Brasileira de Normas Técnicas - ABNT. NBR NM-ISO 2470: papel, cartão e pastas celulósicas: medida do fator de reflectância difusa no azul (alvura ISO). Rio de Janeiro; 200 I.

20 Departamento Nacional de Estradas de Rodagem - DNER. DNER ME 093/94: Solos: determinação da densidade real. Norma Rodoviária. Método de ensaio. Brasília; 1994.

2I Associação Brasileira de Normas Técnicas - ABNT. NBR I 1376: Moinho de bolas: determinação do índice de trabalho: método de ensaio. Rio de Janeiro; 1990.

22 Barbato CN, Sampaio JA. Determinação experimental do Índice de Trabalho (WI). Rio de Janeiro: CETEM-Centro de Tecnologia Mineral; 2007.

23 Figueira HVO, Almeida SLM, Luz AB. Cominuição. In: Luz AB, Sampaio JA, Almeida SLM, editors. Tratamento de Minérios. 4. ed. Rio de Janeiro: CETEM/MCT; 2004, p. I I3-I82.

24 Barrat D, Sherman M. Factors which influence the selection of comminuition circuit. In: Mular AL, Halbe DN, Barratt DJ, editors. Mineral processing plant design, practice and control. SME; 2002. p. 539-565. v. I.

Recebido em: 5 Nov.20I4

Aceito em: 24 Jul. 2015 